\title{
Pseudoparalysis of Marginal Mandibular Nerve Branch by Big Submandibular Gland Sailolith: A Case Report
}

\author{
Kaur R* \\ Department of Otorhinolaryngology, Rajindra Hospital Patiala, India
}

*Corresponding author: Rajwant Kaur, Department of Otorhinolaryngology, BFUHS, Rajindra Hospital Patiala, Punjab, India, Tel: 9501200943; Email: rajwin000@gmail.com
Case Report

Volume 6 Issue 1

Received Date: January 21, 2021

Published Date: February 05, 2021

DOI: $10.23880 /$ ooaj-16000205

\section{Abstract}

Sailolithiasis is the second most common etiology of salivary gland after sialadenitis. Present case of 29 years old male patient with giant submandibular sailolith with stony hard feel below the left angle of the mandible. X ray and CECT neck used for confirmation. Because of big stone incidental finding of pressure paresis of marginal mandibular nerve was noticed. Even patient unaware of that. En-block Submandibular salivary gland excision was done by trans-cervical approach with complete recovery of the nerve within 3-4 post-operative weeks.

Keywords: Submandibular Gland; Marginal mandibular nerve; Pseudoparalysis; Salivary stones; Facial nerve

\section{Introduction}

Salivary gland stone is considered to be the second most common disorder of the salivary gland. Submandibular gland has got highest predilection for sialolithiasis with $80 \%$ occurrence rate, followed by $19 \%$ in the parotid and only $1 \%$ in the sublingual glands [1]. Sialolithiasis is common among the age group of 30 and 60 years, and it is very rare in the paediatric population only $3 \%$ has been reported till now. Males are affected twice as much as females [2]. Usually salivary stones are less than $10 \mathrm{~mm}$ but more than $10 \mathrm{~mm}$ that consider as big in size and has been reported in the literature [3].

Salivary stone may be asymptomatic, or it may presents with painless swelling when duct get obstructed. Patient may present with pain and swelling of the involved gland during meal because saliva production is maximum at this time against a fixed obstruction. Patient may have on and off symptom of episodic swelling and discomfort or symptoms may persist because of salivary fluid accumulation [4]. Salivary stone are very easy to diagnose by its clinical features. On radiographs occlusal view is commonly used to visualise radiopaque stones. Another method is Sialography which is used in patients of sialadenitis related to the radiolucent stones, but it is contradicted in case of acute infection or patients who are allergic to contrast [3]. Ultrasonography having $99 \%$ accuracy and is a gold standard for diagnosis. Other techniques like scintigraphy, CT (contrast and without contrast), and sialoendoscopy are used for the diagnosis of sialolithiasis [5].

Management of salivary stone basically depends upon its location and the symptoms associated with it. It can be broadly divided in two type conservative and surgical. In conservative management sialagogues and gland massage are very useful in flushing the stone out of the duct. In case of sialadenitis antibiotics are given first then simple transoral sialolithotomy done. Bigger stones are fragmented if it is necessary by using an extracorporeal and extracorporeal shock wave lithotripsy or laser assisted sialolithotomy can be used. Other methods are sialoendoscopy and basket retrieval, salivary gland excision etc.

\section{Case Report}

An adult male of 29 years old presented with the history of swelling below left mandible from last 3 years, which was insidious in onset. Initially it was of pea size progressed to walnut size. Swelling was painful and associated with chewing 
of meal. Pain was dull aching, intermittent aggravated by chewing, localized below the left mandible and non-radiating in nature. There was history of sore throat of 3-4 episodes per year. Patient having no history of loss of appetite or excessive salivation. Local examination, on inspection a single globular size mass of approximately $2 \mathrm{~cm} \times 2 \mathrm{~cm}$ in the left submandibular region was present. On palpation, single globular, deep seated, firm to hard non-tender mass with smooth surface, regular margin, mobile of approximately $2.5 \mathrm{~cm} \times 2 \mathrm{~cm}$ present in the left submandibular region. On bimanual palpation mass was felt in the floor of the mouth. Mass was stony hard, mobile not fixed to underline structure. On local examination it was found that patient left lower lip was dropped down during full denture smile. Even patient was unaware of that, it was an incidental and unique finding of this case. As the sailolith was big enough and deep seated probability of pressure on the marginal mandibular nerve branch was high.

Patient routine investigations were normal. X-ray of left submandibular revealed radiopaque shadow of calculus in the submandibular area of approximately $11.6 \mathrm{~mm} \times 20 \mathrm{~mm}$ in size. CECT Neck was advised which confirmed an enlarged left submandibular gland of size $3.2 \mathrm{~cm} \mathrm{X} 2.5 \mathrm{~cm}$ with a hyper dense focus of $1.16 \mathrm{~cm} \mathrm{x} 1.19 \mathrm{~cm}$ seen in the proximal part of the left submandibular duct with extraductal extension maximum diameter of $3.7 \mathrm{~mm}$, another hyper dense focus of $5.4 \mathrm{~mm}$ X $5.4 \mathrm{~mm}$ seen in the distal part of left submandibular duct in sublingual area, tonsillolith $3.5 \mathrm{~mm} \mathrm{X} 2.6 \mathrm{~mm}$ in palatine tonsil in right side (Figure 1). Rest of the salivary glands were visualized to be normal.

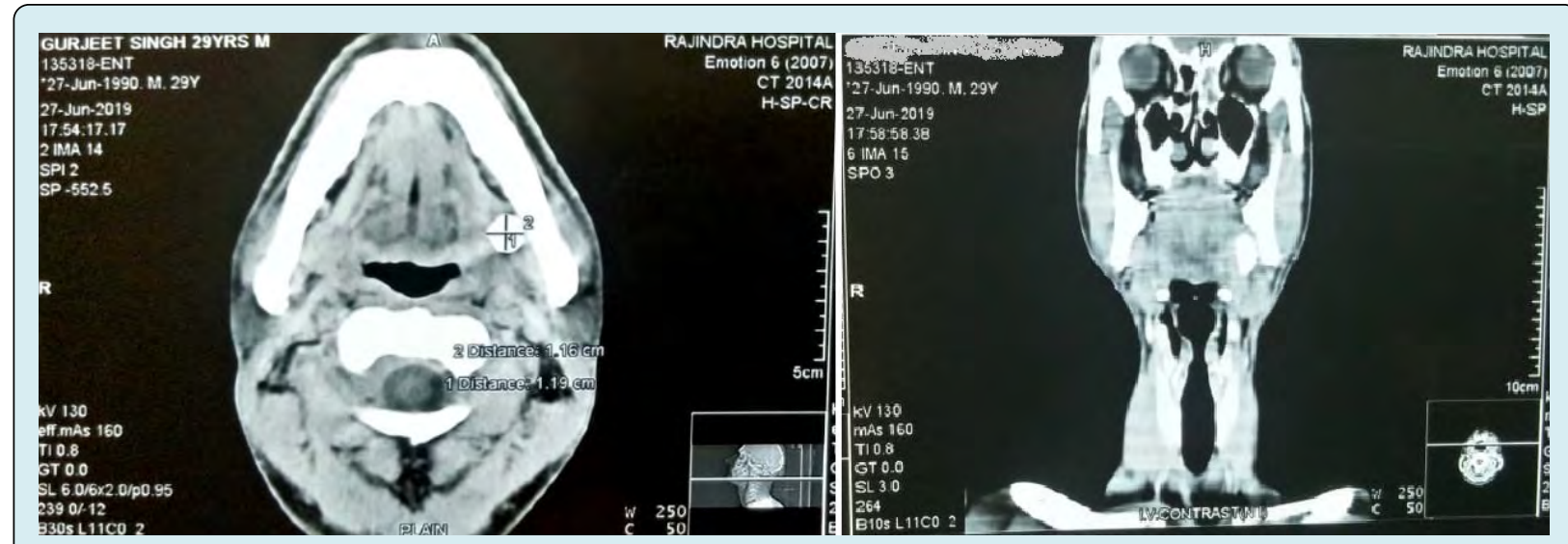

Figure 1: CT scan axial and coronal cuts showing hyper dense shadow in the left submandibular region.

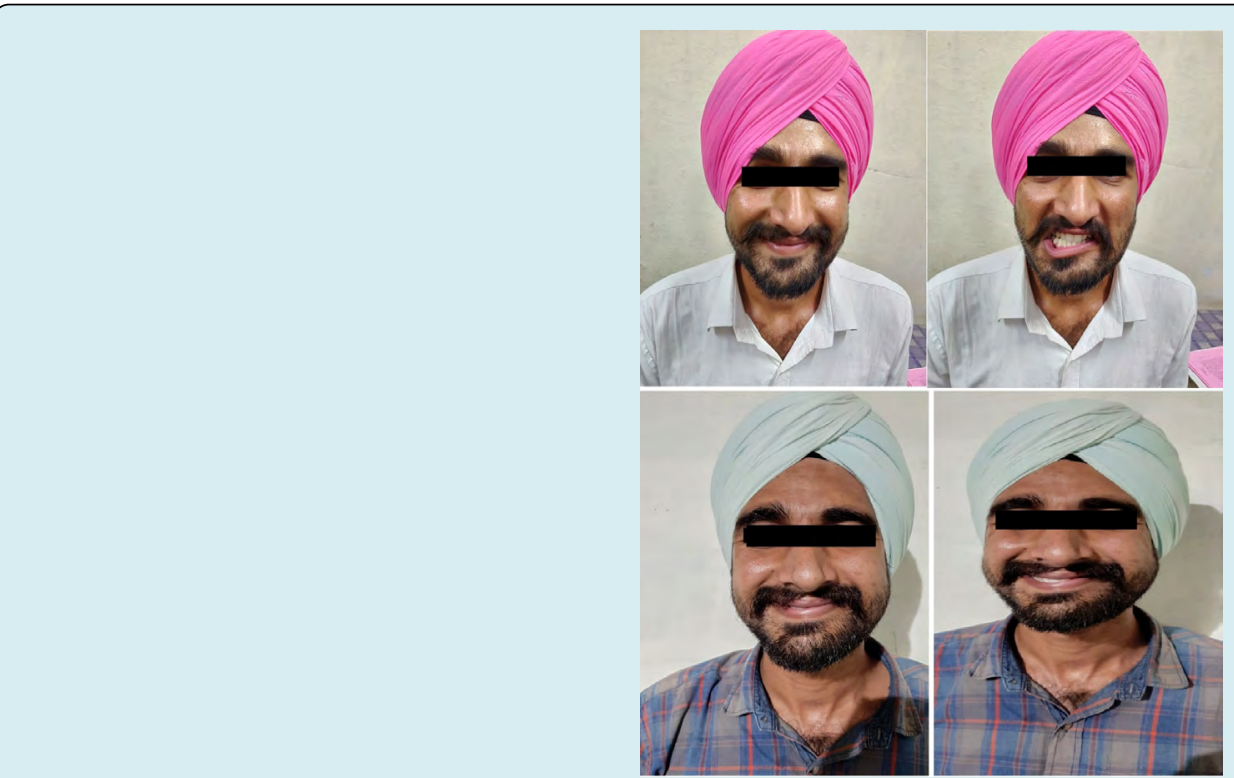

Figure 2: Pre-operative (above) and Post-operative picture after 4 weeks (below) showing Zygomaticus type smile (Left) and near normal denture smile (right). 


\section{Otolaryngology Open Access Journal}

Patient was operated under general anesthesia. Gland was freed along with Wharton's duct, specimen was delivered out and sent for histopathological examination which was later found to be normal glandular tissue. No malignant cells were seen. Saline irrigation was done after achieving heamostasis. Wound was closed in two layer with vicryl 3'0 and nylon 3'0. A Drain was placed. At Four weeks follow up lower lip depressor function on left side was found to be near normal (Figure 2).

\section{Discussion}

The submandibular gland is most commonly affected because of its anatomic location, tortuous long duct with a narrow orifice as compare to the main portion of the duct. The distal stones can also be expressed and manipulated through the orifice of the duct with the aid of dilating lacrimal probes if required to open the duct. Whereas the proximal stones residing in the hilum of the gland or within the gland, such stones are usually removed surgically through a transcervical excision [6,7]. The transoral and transcervical have their own merits and demerits. Transcervical procedure being traditionally old method with less morbidity, involves the preservation of three nerves: the marginal mandibular branch of facial nerve, lingual nerve and the hypoglossal nerve. On the other hand transoral has better cosmetic result with less hospital stay with comparatively less chances of injuries to marginal mandibular nerve. The reported incidence of its injury is $0 \%$ to $20 \%$ incidence even in the experienced surgeon hands [8]. Ellenbogen mentioned two cases of transient "pseudoparalysis of the marginal mandibular nerve" as a result of injury to cervical branch of facial nerve; it was distinguished from marginal mandibular nerve injury as the patient can still evert the lower lip due to the intact functioning of mentalis muscle [9]. Cervical branch injury is associated with an excellent prognosis, with complete recovery within 3 week to 6 month on average. But the present case presented with the pressure paralysis due to stone on the left side marginal mandibular branch of the facial nerve, resulting in the paralysis of the depressor angulioris muscles and depressor labii inferioris muscles, which manifested as lower lip depressor dysfunction on left side and surprisingly patient was unaware of that so the exact duration could not be make out.

It was further confirmed by asymmetrical full denturetype smile and zygomaticus type smile which usually didn't manifest the disability. To our knowledge this the first case of submandibular gland duct stone that manifested like that, otherwise it usually occur during other surgical procedures like rhytidectomy, parotidectomy, carotid endarterectomy, open reduction of mandibular angle fracture, submental flap harvesting, and intentionally during neck dissection for cancer clearance. As the nerve is highly vulnerable to injury in the submandibular triangle because of its highly variable anatomy that cannot be ensured by anatomic landmarks [10]. In present report surgical excision of the gland with stone removed the pressure from nerve and it recovered conservatively in 3-4 week postoperative period.

\section{Conclusion}

So unusual size large sailolith is a rare finding commonly seen in the submandibular salivary gland. To our knowledge it is the first case report on the incidental finding of pressure paralysis of the marginal mandibular nerve branch due to giant sailolith and which was managed successfully after excision of gland with stone by transcervical approach.

\section{Acknowledgments}

I wish to thank Dr. Pawan Kumar Kamal, Consultant AP Healthcare Patiala, who has helped me prepare this case report.

\section{References}

1. Batori M, Mariotta G, Chatelou H, Casella G, Casella MC (2005) Diagnostic and surgical management of submandibular gland sialolithiasis: Report of a stone of unusual size. Eur Rev Med Pharmacol Sci 9(1): 67-68.

2. Siddiqui SJ (2002) Sialolithiasis: An unusually large submandibular salivary stone. Br Dent J 193(2): 89-91.

3. Isacsson $\mathrm{G}$, Isberg $\mathrm{A}$, Haverling $\mathrm{M}$, Lundquist $\mathrm{PG}$ (1984) Salivary calculi and chronic sialoadenitis of the submandibular gland: A radiographic and histologic study. Oral Surg Oral Med Oral Pathol 58(5): 622-627.

4. Manjunath R, Burman R (2009) Gaint submandibular sialolith of remarkable size in the common area of Wharton's Duct: a case report. J Oral Maxillofac Surg 67(6): 1329-1332.

5. Marchal F, Kurt AM, Dulguerov P, Lehmann W (2002) Submandibular diagnostic and interventional sailendoscopy: new procedure for ductal disorders. Ann Otol Rhinol Laryngol 111(1): 27-35.

6. Bayindir T, Cetinkaya Z, Toplu Y, Akarcay M (2012) A Giant Submandibular Sialolithiasis that Erupted Spontaneously to the Mouth: A Case Report. JIUMF 19: 188-191.

7. Marwaha M, Nanda KD (2012) Sialolithiasis in a 10 year old child. Indian J Dent Res 23(4): 546-549.

8. Ichimura K, Ki N, Tanaka T (1997) Nerve paralysis after surgery in the submandibular triangle: review 
of University of Tokyo Hospital experience. Head Neck 19(1): 48-53.

9. Ellenbogen R (1979) Pseudo-paralysis of the mandibular branch of the facial nerve after platysmal face-lift operation. Plast Reconstr Surg 63(3): 364.

10. Khanfour AA, Metwally ESAM (2014) Marginal mandibular branch of the facial nerve: an anatomical study. Alexandria Med J 50(2): 131-138. 